\title{
Building a doped Mott system by holography
}

\author{
Yi Ling ${ }^{1,2},{ }^{*}$ Peng Liu ${ }^{1}{ }^{\dagger}$ Chao Niu ${ }^{1}, \ddagger$ and Jian-Pin $\mathrm{Wu}{ }^{3,2 \S}$ \\ 1 Institute of High Energy Physics, \\ Chinese Academy of Sciences, Beijing 100049, China \\ 2 State Key Laboratory of Theoretical Physics, Institute of Theoretical Physics, \\ Chinese Academy of Sciences, Beijing 100190, China \\ ${ }^{3}$ Institute of Gravitation and Cosmology, \\ Department of Physics, School of Mathematics and Physics, \\ Bohai University, Jinzhou 121013, China
}

\begin{abstract}
We construct a holographic model in the framework of Q-lattices whose dual exhibits metalinsulator transitions. By introducing an interacting term between the Q-lattice and the electromagnetic field in bulk geometry, we find such kind of transition can be Mott-like. The evidences are presented as follows. i) The transition from a metallic phase to an insulating phase occurs when the lattice constant becomes larger. ii) A hard gap in the insulating phase can be manifestly observed in the optical conductivity. Nevertheless, in the zero temperature limit this model exhibits novel metallic behavior, featured by a gap as well as a zero-frequency mode with tiny spectral weight. It implies that our model is dual to a doped Mott system in one dimension where umklapp scattering is frozen at zero temperature. The similarity between this model and some organic linear chain conductors is briefly discussed.
\end{abstract}

*Electronic address: lingy@ihep.ac.cn

${ }^{\dagger}$ Electronic address: liup51@ihep.ac.cn

‡Electronic address: niuc@ihep.ac.cn

§Electronic address: jianpinwu@mail.bnu.edu.cn 


\section{INTRODUCTION}

Gauge/gravity duality has provided powerful tools for investigating strongly correlated systems in condensed matter physics. In particular, the metal-insulator transition has recently been implemented in this approach, in which the translational symmetry is broken by various lattice structures [1-6]. However, until now it is still challenging to construct a holographic model describing Mott insulator, which plays a crucial role in a strongly correlated electron system and is always responsible for the building of high $T_{c}$ cuprate superconductors [7]. In this paper we intend to construct such a holographic model whose dual can be viewed as a doped Mott system.

The notion of a Mott insulator was originally proposed by Mott in the 1930s [8]. In his thought experiment, a metal could become an insulator when increasing the lattice constant, simply because the ability of electrons hopping from one site to its neighbors decays. Mott's idea has been confirmed in experiments (for instance, see [9-11] and references therein.), in which the metal-insulator transition occurs when changing external parameters such as the pressure which leads to the change of lattice spacing. In the theoretical aspect, the Mott thought experiment can be formalized in the Hubbard model [12]. The Hamiltonian of Hubbard model contains two key ingredients. One is the kinetic energy which describes the electron hopping process and the other one is the potential energy which describes onsite Coulomb interaction between two electrons with opposite spin. Roughly speaking, the electron hopping process is responsible for the metallic behavior while the repulsive Coulomb interaction is for the insulating state. When the kinetic and potential energies are of the same order, it is extremely difficult to solve the Hubbard model exactly except for the onedimensional case. A powerful technique for solving the Hubbard model is the dynamical mean field theory (DMFT) $[13,14]$, in which the lattice model is replaced by an effective single impurity model.

In the holographic approach, dynamically generating a Mott gap has been proposed in [15-17] by considering fermions with dipole coupling over an AdS-Reissner-Nordström (AdS-RN) geometry ${ }^{1}$. However, such a Mott gap is interaction driven and achieved only in the probe limit. It should be stressed that the bulk geometry of the background is

\footnotetext{
${ }^{1}$ It is worthwhile to notice that the dual boundary theory of a holographic system with dipole coupling fermions may suffer from superluminal modes in large momentum limit [18] .
} 
completely dual to a metallic phase because of the IR fixed point being $\mathrm{AdS}_{2}$. Later, novel metal-insulator transitions have been implemented in black hole backgrounds with lattice structure. The key point in this framework is to make the near horizon geometry unstable such that the IR fixed point can deform from $\mathrm{AdS}_{2}$ to other saddle points which may be dual to an insulating phase [1-3]. In this paper we intend to push this approach forward and explicitly construct a gravitational dual model which exhibits the fundamental features of a one-dimensional doped Mott system. Specifically, we will introduce an interacting term between the Q-lattice and the electromagnetic field in bulk geometry. It turns out that the transition from a metallic phase to an insulating phase occurs when the lattice constant becomes larger, which explicitly visualizes the thought experiment proposed by Mott in a holographic manner. More importantly, the linear perturbation analysis will demonstrate that a hard gap can be manifestly observed in the optical conductivity ${ }^{2}$. Nevertheless, we find this model always contains nonvanishing zero-frequency modes. Although its spectral weight is a tiny proportion of the total, it is responsible for the large metallic conductivity such that the system exhibits a novel metallic behavior in zero temperature limit. This behavior implies that our model is a doped system where umklapp scattering is frozen at zero temperature. Interestingly enough, we find our model is analogous to some organic linear chain conductors which are featured by a gap as well as nonvanishing Drude peak in metallic state $[19,20]$. We will briefly address this issue in the end of our paper.

\section{THE HOLOGRAPHIC SETUP}

Our holographic setup is based on a Q-lattice structure, which has originally been proposed in [2] and subsequently investigated in literature [3, 5, 17, 21, 22]. Such a gravitational dual model contains a complex scalar field and a $U(1)$ gauge field. We introduce the action as

$$
S=\frac{1}{2 \kappa^{2}} \int d^{4} x \sqrt{-g}\left[R+6-\frac{V(\Phi)}{4} F^{\mu \nu} F_{\mu \nu}-|\nabla \Phi|^{2}-m^{2}|\Phi|^{2}\right],
$$

where we have fixed the $A d S$ length scale $L=1 . \Phi$ is uncharged and will be responsible for the breaking of the translational symmetry. Comparing with the original action proposed

\footnotetext{
${ }^{2}$ Near the completion of this manuscript, we noticed the appearance of [23] in which an insulator with a hard gap has also been implemented in a holographic manner.
} 
in [2], we have introduced a new coupling $V(\Phi)$ between the scalar field and the gauge field, which will play a crucial role in generating a Mott-gap in insulating phase. For explicitness, in this paper we will set $V(\Phi)=1-\beta|\Phi|^{2}$ with a positive parameter $\beta^{3}$. Such a term is similar to that in [24]. Obviously, the above construction goes back to the original form in [2] if one sets $\beta=0$. Therefore, next we will skip all the technical steps for constructing a Q-lattice background but just present the results.

The ansatz for a background which has a Q-lattice structure only along $x$ direction is given by

$$
\begin{aligned}
d s^{2} & =\frac{1}{z^{2}}\left[-(1-z) p(z) U d t^{2}+\frac{d z^{2}}{(1-z) p(z) U}+V_{1} d x^{2}+V_{2} d y^{2}\right] \\
A & =\mu(1-z) \psi d t \\
\Phi & =e^{i k x} z^{3-\Delta} \phi
\end{aligned}
$$

where $\mu$ is the chemical potential, $p(z)=1+z+z^{2}-\mu^{2} z^{3} / 4$ and $\Delta=3 / 2+\left(9 / 4+m^{2}\right)^{1 / 2}$. All the functions $U, V_{1}, V_{2}, \psi$ and $\phi$ depend on the radial coordinate $z$ only. Through this paper we set $m^{2}=-2$. For a given $\beta$, each electrically charged black hole solution with Qlattice is specified by three scaling-invariant parameters, namely, the Hawking temperature $T / \mu$ which is given by $\left(12-\mu^{2}\right) U(1) /(16 \pi \mu)$, the lattice amplitude $\lambda / \mu^{3-\Delta}$ where $\lambda \equiv \phi(0)$ and the wave vector $k / \mu$. For convenience, we abbreviate these quantities to $T, \lambda$ and $k$, respectively. Moreover, it is worthy to point out that in this formalism the background has a periodic structure manifestly such that $a \equiv 2 \pi / k$ can be understood as the lattice constant of the dual model, which is important for us to link this holographic scenario with the thought experiment by Mott in next section.

\section{PHASE DIAGRAM AND OPTICAL CONDUCTIVITY}

It is first shown in [2] that the dual system of a Q-lattice background exhibits metalinsulator transitions when varying the lattice parameters $\lambda$ and $k$, which corresponds to the special case with $\beta=0$ in our model. When the parameter $\beta$ is turned on we find that the coupling term will play the role of generating a hard gap for the optical conductivity, which

\footnotetext{
${ }^{3}$ We require that $\beta$ is positive such that the system is always stable under linear perturbations. Otherwise a negative $\beta$ would induce the instability of near horizon geometry, leading to the spontaneous breaking of the translational symmetry at low temperatures, as discussed in $[4,25]$.
} 


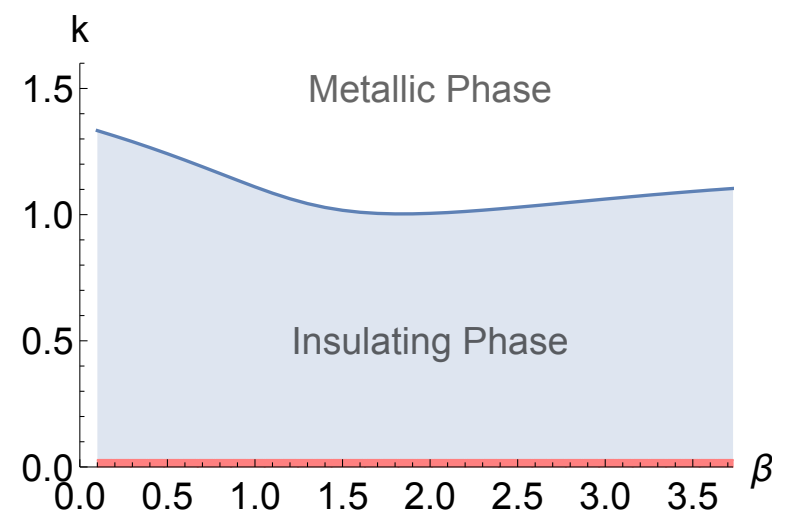

FIG. 1: The phase diagram with $(\lambda=2, T=0.2)$. Our numerical data are collected beyond the pink region with $k<0.03$.

is analogous to the effect of Coulomb interaction $U$ in Mott-Hubbard model. We intend to discuss the phase diagram and the optical properties of the system in following subsections.

\section{A. Finite temperature region}

In this subsection we investigate the $(\beta, k)$ phase diagram and the optical properties of the dual system when the temperature is not too low. We will demonstrate that a metalinsulator transition occurs when the lattice constant becomes larger. Furthermore, a Mott hard gap can be manifestly observed in the plot of the optical conductivity in insulating phase when the parameter $\beta$ is adjusted to an appropriate value.

First, we plot the $(\beta, k)$ phase diagram at the temperature $T=0.2$, as illustrated in Fig.1. The insulating phase corresponds to $\sigma_{D C}^{\prime}(T)>0$ while the metallic phase $\sigma_{D C}^{\prime}(T)<0$. Thus the critical line is described by $\sigma_{D C}^{\prime}(T)=0$. $D C$ conductivity can be obtained by either the zero-frequency limit of optical $A C$ conductivity, or the numerical analysis on the horizon with formula $\sigma_{D C}=\left.\sqrt{\frac{V_{2}}{V_{1}}}\left[V(\Phi)+\frac{V_{1}}{2}\left(\frac{V(\Phi) \mu \psi}{k \phi}\right)^{2}\right]\right|_{z=1}$. In this phase diagram, one can easily find that a metal-insulator transition occurs when the wave number deceases (or the lattice constant increases) for a given lattice amplitude $\lambda$ and coupling parameter $\beta$, which could be viewed as a holographic realization of Mott thought experiment. Therefore, the $(\beta, k)$ phase diagram provides us the first evidence that the resulting metal-insulator transition in our model is Mott-like, which is induced by changing the lattice constant. 



FIG. 2: The real part (blue curves) and the imaginary part (brown curves) of optical conductivity with different values of $\beta$ (the left plot is for $\beta=0$ while the right one for $\beta=3$ ). The other parameters are fixed as $\lambda=2, k=0.03$ and $T=0.2$. The insets in both plots are the blow-up of the optical conductivity in low frequency region.

Next we provide the second key evidence for the Mottness of this sort of insulators by a linear perturbation consideration. We compute the optical conductivity as a function of frequency when applying an external electric field along $x$ direction. In holographic setup this can be achieved by introducing the following self-consistent perturbations over the background,

$$
\delta g_{t x}=h_{t x}(t, z), \quad \delta A_{x}=a_{x}(t, z), \quad \delta \Phi=i e^{i k x} z^{3-\Delta} \phi(t, z)
$$

where the time dependence are all set as $e^{-i \omega t}$, such that the optical conductivity is given by $\sigma(\omega)=\left.\frac{\partial_{z} a_{x}(z)}{i \omega a_{x}(z)}\right|_{z=0}$.

We explicitly demonstrate that a hard gap can be produced in insulating phases with the increase of the parameter $\beta$, as illustrated in Fig.2. The left plot in Fig.2 is for $\beta=0$, only a soft gap being observed. Previously, a similar phenomenon has also been presented in $[1,2]$. While the right plot of Fig.2 is for $\beta=3$, in which a hard gap can be obviously seen. It is one of the important characteristics of a class of Mott insulators. In particular, it is usually believed that in the Mott-Hubbard model, the formation of a hard gap is due to the localization of electrons driven by the Coulomb interaction $U$. In previous holographic insulator models, only a soft gap is observed in the optical conductivity [1-3]. Here we provide a new localization mechanism for the gap formation by holography. In this mechanism, the coupling parameter $\beta$ plays a crucial role, which is similar to the Coulomb interaction $U$. In addition, we would like to present the following remarks on the optical conductivity when we change the value of the coupling parameter $\beta$. 
- The gap becomes more evident with the increase of $\beta$. This phenomenon is also in accordance with the effect of increasing $U$ in Mott-Hubbard model since the enhancing strength of electron-electron interaction will localize electrons. If one extracts the charge density $\rho$ from the time component of the gauge field in background solutions, it is also found that the charge density decreases with the increase of $\beta$, which possibly provides an understanding on the suppression of the optical conductivity and the emergence of a hard gap.

- The insets in Fig.2 illustrate the low frequency behavior of the optical conductivity. It turns out that generically the low frequency behavior can be fit well with the Drude formula. This phenomenon asks for further understanding since now the dual system lies in the insulating phase. Moreover, comparing two insets in Fig.2 we obviously see that the Drude weight diminishes with the increase of $\beta$.

- With the increase of $\beta$, more resonance peaks show up in the intermediate frequency region of the optical conductivity and approximately exhibit a periodic distribution (as is seen in the right plot in Fig.2), indicating a multiband structure for the dual theory.

Next, we study the properties of our model in zero temperature limit.

\section{B. In zero temperature limit}

To explore the characteristics of this system thoroughly, we cool it to extremely low temperature. As an example, we show the optical conductivity at $T=0.004$ in Fig.3, which is in an insulating phase. It is noticed that the appearance of a hard gap as well as resonances resembles the phenomena in the second plot of Fig.2 except that the resonance peaks become sharper. Moreover, the low frequency behavior of the optical conductivity (shown in the inset) can be well fit with Drude formula as well.

In order to explore the quantum critical behavior of the system, we intend to study the structure of phase diagram in zero temperature limit. For definiteness, we plot the $(\beta, T)$ phase diagram with $\lambda=2$ and $k=0.03$, as shown in Fig.4. We present our remarks on this phase diagram in order as follows. 


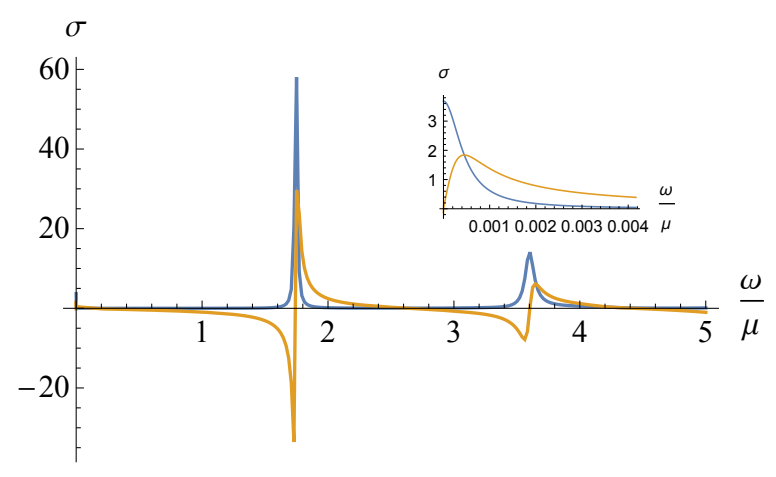

FIG. 3: The real part (blue curves) and the imaginary part (brown curves) of optical conductivity for $\lambda=2, k=0.03, \beta=0.766$ and $T=0.004$. The inset is the low frequency behavior of the conductivity.

- The phase diagram is divided into three regions corresponding to the metallic phase, insulating phase and no-solution region, respectively. The critical line between the metallic phase and the insulating phase is determined by $\sigma_{D C}^{\prime}(T)=0$. Numerically, we find the charge density $\rho$ is always decreasing with the increase of $\beta$, and we show an example in Fig.5. As $\rho$ becomes vanishing, no numerical solution to background equations could be found when further increasing the parameter $\beta$, therefore a nosolution region forms as shown in Fig.4. The interface between the no-solution region and the other two regions can roughly be described by $\rho \simeq 0$. From the viewpoint of dual field theory, the fact of $\rho \rightarrow 0$ might correspond to $U \rightarrow \infty$ in Mott-Hubbard model since in this limit all the electrons will be localized. Moreover, we point out that the coupling term $V(\Phi)$ should be positive everywhere to avoid ghosty gauge fluctuations. We have checked the behavior of $V(\Phi)$ over all the $z$-axis and found that in usual cases it is always positive. When $\beta$ becomes large and particularly points to the borderline of no-solution region, $V(\phi)$ approaches to but never below 0 in a small region of $z$. To guarantee our model is safe we have also checked the case for $V(\Phi)=\left(1-\beta|\Phi|^{2}\right)^{2}$ which is positive definite, and we qualitatively recovered all the results and phenomena in the current paper.

- Numerically we have found that the hard gap is not evident until the parameter $\beta$ is adjusted to a relatively large value $\beta_{c}$. In Fig.4, for parameters $\lambda=2$ and $k=0.03$ we find this critical value is about $\beta_{c} \simeq 0.4$. 
- We are very concerned with the intersecting part of these three regions in zero temperature limit because it provides us a hint on whether the system could fall into an insulating region with hard gap at zero temperature. Therefore, we blow up the intersecting region as shown in the inset of Fig.4. Unfortunately from this inset we notice that there is no such an insulating region in the zero temperature limit.

- To demonstrate the metal-insulator transition in a more transparent manner, we plot the DC resistivity $\rho_{\mathrm{DC}}$ as a function of temperature in the left plot of Fig.6, with parameters specified by the purple line in Fig.4. It is observed that the $\rho_{\mathrm{DC}}$ rises up with the decreasing temperature at first, but after reaching its maximum it turns to go down and eventually falls into zero when approaching the zero temperature. Interesting enough, this behavior is much similar to the one as described in [26] when the system is close to the Mott transition.

- The low frequency behavior of the optical conductivity can always be fit well with the Drude formula whenever a hard gap is evidently observed. However, the Drude weight is always diminishing as the temperature decreases. For explicitness, we show the low frequency region of the real part of conductivity at temperatures $T=0.006$ and $T=0.004$ in the right plot of Fig.6. In this figure the decrease of the Drude weight with the temperature is manifest. We find this tendency is also true for a metallic phase. That is to say, although the DC conductivity becomes larger with the decrease of the temperature in metallic phases, the Drude weight is always diminishing. Nevertheless, the Drude weight does not vanish in the zero-temperature limit, whatever the phase is. This phenomena could be reflected as the feature of a doped Mott system or a deviation from the commensurate filling in one dimension as we will elaborate below.

- Finally, we intend to claim that all above observations do not change qualitatively with other choices of parameters $(\lambda, k)$. For instance, we may have a larger value of $\lambda$, then the metallic region may shrink into a smaller region and the critical value $\beta_{c}$ for an evident hard gap could be smaller, while at the same time one would find the no-solution region expands such that the insulating phase with hard gap remains absent in zero temperature limit.

In the end of this section we intend to focus on an important issue related to understanding 


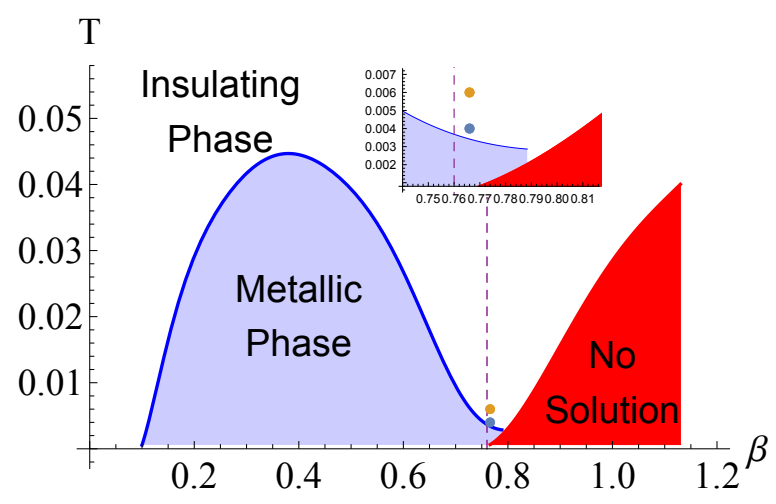

FIG. 4: $(\beta, T)$ phase diagram with $\lambda=2, k=0.03$. The inset is a blow up of the intersecting region nearby $\beta=0.78, T=0.003$. The purple dashed line and two dots denote the parameters adopted in Fig.6.

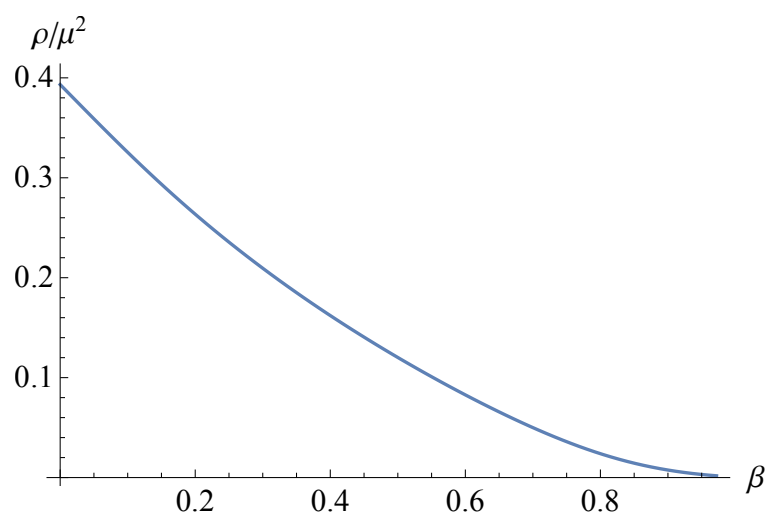

FIG. 5: Charge density $\rho / \mu^{2}$ as a function of $\beta$ at $k=0.03, \lambda=2, T=0.03$.

the emergence of the metallic phase region in zero temperature limit, as illustrated in Fig.4. Theoretically, the presence of a gap as well as a tiny spectral weight in low temperature limit is in accordance with a doped Mott system as described in [27, 28]. First, in high frequency region there are interband transitions and the doping will not affect the system. Thus, the high frequency behavior will be the same as the commensurate filling. However, for the frequencies being smaller, the interband transitions are blocked, and then the conductivity becomes vanishing, leading to the formation of a gap. Second, when a system is doped, the Fermi energy may not fall into the gap region such that intraband transitions are still possible and result in nonzero low frequency excitations, which is supposed to be responsible for the metallic behavior of the system. Though the Drude weight is very tiny in the spectrum, its nonvanishing feature could give rise to a divergent DC conductivity in zero 

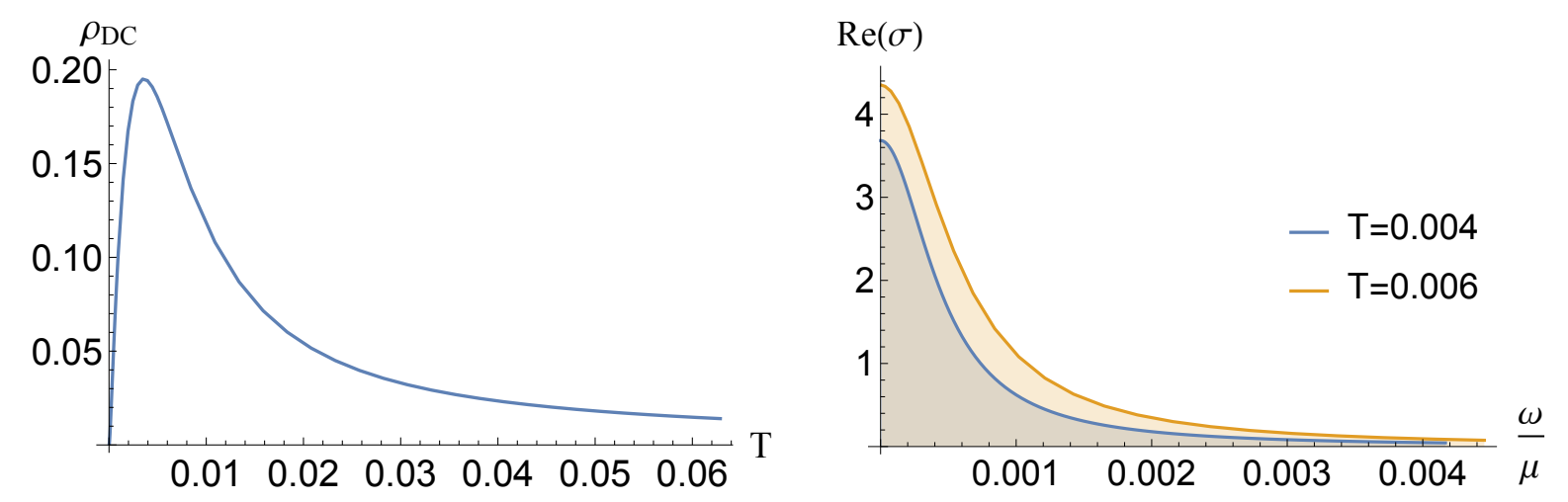

FIG. 6: The left plot is for the temperature dependence of DC resistivity $\rho_{\mathrm{DC}}$ at extremely low temperature region for the selected $\lambda=2, k=0.03, \beta=0.76$ (which corresponds to the purple dashed line in Fig.4.). The right plot shows the real part of optical conductivity at temperatures $T=0.004$ and 0.006 , with $\lambda=2, k=0.03, \beta=0.766$ (which are marked as the dots in the inset of Fig.4).

temperature limit. From a microscopic point of view, this phenomenon could be understood as a consequence of doping which deviates the system from commensurate filling such that the umklapp scattering is frozen in a one dimensional system.

The gapped behavior as well as a tiny Drude weight of optical conductivity have been observed in some organic materials such as the TMTSF family [19, 20], which is analogous to a doped Mott system [27]. The organic conductors are quasi-one dimensional systems with a perpendicular hopping between chains. It is also argued in [27] that doping could be attributed to the transverse hopping between chains, which results in deviation from commensurate filling because of the warping of the Fermi surface. Nevertheless, we need to point out that for such organic conductors the metallic behavior of the zero frequency modes due to the interchain transitions are always decoherent and deviated from a Drude law [19], which seems in contrast to what we observed here.

\section{DISCUSSION}

In this paper we have constructed a holographic model based on Q-lattice which can be dual to a doped Mott system. The key ingredient in our proposal is the introduction of a coupling term between the Maxwell field and the scalar field in the Q-lattice geometry. 
We have explicitly demonstrated that the Mott thought experiment can be visualized by holography in Q-lattice framework. More importantly, a hard gap can be produced in the optical conductivity when the coupling parameter $\beta$ is relatively large. While in the zero temperature limit we find the Drude weight is not vanishing and the system exhibits a novel metallic behavior. All above features are closely similar with those of a doped Mott system in one dimension, and have been observed in the TMTSF family of organic materials.

Our results reported here are preliminary and should be thought of as the first step for building a holographic model for Mott insulator which is usually commensurate. To gain more insight into this topic, a lot of work needs to be done. Especially, the following crucial issues should be explored further. First, it is very desirable for one to understand why the coupling term plays the role of generating a hard gap for the optical conductivity, which is very crucial for us to figure out the direct connection between the coupling term in this holographic model and the electron-electron interaction rooted in a realistic Mott system. We expect an analytical investigation on the current-current correlator and the deformation of the near horizon geometry like [29-31], would be helpful for us to understand how the coupling term changes the gapless geometry to gapped one, as well as the multiband structure and the emergence of novel metallic phase in the zero temperature limit. Alternatively, one could provide more evidences for doping if the spectral weight transfer could be found in the fermionic spectral function including an appropriate coupling between fermions and the lattice background. Second, we are crying for some new mechanism in Q-lattice framework to suppress the Drude weight so as to achieve an insulating phase with a hard gap in the zero temperature limit. Finally we remark that throughout this paper we have only considered Q-lattice structure in one spatial direction. It is very worthwhile to investigate the Mottness of the dual system in two or higher dimensional Q-lattice background or other lattice models [32]. Our work in this direction is under progress.

\section{Acknowledgments}

We are grateful to Matteo Baggioli, Aristomenis Donos, Jerome Gauntlett, Blaise Goutéraux, Sean Hartnoll, Honggang Luo, Philip Phillips, Jie Ren, Yu Tian, Yidun Wan, Yuan Wan, Zhuoyu Xian, Yifeng Yang, Hongbao Zhang for helpful discussions. We also thank the anonymous referee for detailed comments and helpful suggestions. This work is 
supported by the Natural Science Foundation of China under Grants No. 11275208, No. 11305018 and No. 11178002. Y.L. also acknowledges the support from Jiangxi young scientists (JingGang Star) program and 555 talent project of Jiangxi Province. J. P. Wu is also supported by the Program for Liaoning Excellent Talents in University (No. LJQ2014123). This research was supported in part by Perimeter Institute for Theoretical Physics. Research at Perimeter Institute is supported by the Government of Canada through Industry Canada and by the Province of Ontario through the Ministry of Economic Development and Innovation.

[1] A. Donos and S. A. Hartnoll, Nat. Phys. 9, 649 (2013) [arXiv:1212.2998[hep-th]].

[2] A. Donos and J. P. Gauntlett, JHEP 1404, 040 (2014) [arXiv:1311.3292 [hep-th]].

[3] A. Donos and J. P. Gauntlett, JHEP 1406, 007 (2014) [arXiv:1401.5077 [hep-th]].

[4] Y. Ling, C. Niu, J. Wu, Z. Xian and H. Zhang, Phys. Rev. Lett. 113, 091602 (2014) [arXiv:1404.0777 [hep-th]].

[5] A. Donos, B. Goutéraux and E. Kiritsis, JHEP 1409, 038 (2014) [arXiv:1406.6351 [hep-th]].

[6] M. Baggioli and O. Pujolas, arXiv:1411.1003 [hep-th].

[7] P. A. Lee, N. Nagaosa, X. G. Wen, Rev. Mod. Phys. 78 17-85 (2006), [arXiv:condmat/0410445].

[8] N. F. Mott, Proc. Phys. Soc. London, Ser. A 49, 72 (1937).

[9] M. Imada, A. Fujimori, and Y. Tokura, Rev. Mod. Phys. 70, 1039 (1998).

[10] P. Limelette, P. Wzietek, S. Florens, A. Georges, T.A. Costi, C. Pasquier, D. Jerome, C. Meziere, P. Batail, Phys. Rev. Lett. 91, 016401 (2003), [cond-mat/0301478].

[11] N. Drichko, M. Dressel, C. A. Kuntscher, A. Pashkin, A. Greco, J. Merino and J. Schlueter, Phys. Rev. B 74, 235121 (2006).

[12] J. Hubbard, Proc. Roy. Soc. London A 276, 238. 8, 10 (1963).

[13] W. Metznerand, D. Vollhardt, Phys. Rev. Lett. 62, 324 (1989).

[14] A. Georges, G. Kotliar, W. Krauth, and M. J. Rozenberg, Rev. Mod. Phys. 68, 13 (1996).

[15] M. Edalati, R. G. Leigh, P. W. Phillips, Phys. Rev. Lett. 106 (2011) 091602, [arXiv:1010.3238].

[16] M. Edalati, R. G. Leigh, K. W. Lo and P. W. Phillips, Phys. Rev. D 83, 046012 (2011) [arXiv:1012.3751 [hep-th]]. 
[17] Y. Ling, P. Liu, C. Niu, J. P. Wu and Z. Y. Xian, JHEP 1412, 149 (2014) [arXiv:1410.7323 [hep-th]].

[18] M. Kulaxizi and R. Rahman, arXiv:1507.08284 [hep-th].

[19] M. Dressel et al., Phys. Rev. Lett. 77, 398 (1996).

[20] V. Vescoli et al., Science 281, 1181 (1998).

[21] Y. Ling, P. Liu, C. Niu, J. P. Wu and Z. Y. Xian, JHEP 1502, 059 (2015) [arXiv:1410.6761 [hep-th]].

[22] Y. Ling, P. Liu, C. Niu, J. P. Wu and Z. Y. Xian, arXiv:1502.03661 [hep-th].

[23] E. Kiritsis and J. Ren, arXiv:1503.03481 [hep-th].

[24] E. Mefford and G. T. Horowitz, Phys. Rev. D 90, no. 8, 084042 (2014) [arXiv:1406.4188 [hep-th]].

[25] A. Donos and J. P. Gauntlett, Phys. Rev. D 87, no. 12, 126008 (2013) [arXiv:1303.4398 [hep-th]].

[26] A. Georges, S. Florens, T.A. Costi, Journal de Physique IV 114, 165 (2004), [arXiv:condmat/0311520].

[27] T. Giamarchi, Physica B 230-232 975 (1997)

[28] T Giamarchi, Quantum Physics in One Dimension, (Clarendon Press, 2003)

[29] C. Charmousis, B. Gouteraux, B. S. Kim, E. Kiritsis and R. Meyer, JHEP 1011, 151 (2010) [arXiv:1005.4690 [hep-th]].

[30] B. Goutraux, JHEP 1404, 181 (2014) [arXiv:1401.5436 [hep-th]].

[31] R. A. Davison and B. Goutraux, arXiv:1505.05092 [hep-th].

[32] M. Rangamani, M. Rozali and D. Smyth, JHEP 1507, 024 (2015) [arXiv:1505.05171 [hep-th]]. 